\title{
Gogi aphasia or semantic dementia? Neuropsychological evidence for an amodal, dynamic semantic system
}

\author{
Matthew A. Lambon Ralph ${ }^{\mathrm{a}}$ and Karalyn Patterson ${ }^{\mathrm{b}}$ \\ ${ }^{a}$ University of Manchester, UK \\ ${ }^{\mathrm{b}}$ MRC Cognition and Brain Sciences Unit, Cambridge, UK
}

Some patients with progressive fluent aphasia present with poor verbal comprehension and profound word-finding difficulties in the context of much better picture comprehension and object use. The Japanese term Gogi (literally "word-meaning") aphasia matches this behavioural pattern. The alternative label of semantic dementia is most often used for these patients and this term emphasises a generalised degradation of conceptual knowledge that encompasses both verbal and non-verbal comprehension. This difference in terminology is mirrored by two opposing views of how conceptual knowledge is represented. One approach argues that dissociations between verbal and non-verbal comprehension reflect separate, modality-specific semantic systems. The alternative approach provides an explanation in terms of impairment to a unitary semantic system within a framework that emphasises the underlying differences in the mapping between surface form and meaning. The same idea can be found in classic neurological accounts and contemporary theories of semantic memory, including some implemented PDP models. These theories assume that concepts are acquired through a learning process that draws together information experienced in each of our verbal and non-verbal modalities. These accounts embody three critical assumptions: semantic representations are amodal; the mapping between surface form and meaning varies for different modalities; and the representations are dynamic.

Patients with semantic dementia provide an excellent patient model with which to test these opposing accounts because the conceptual impairment is both selective (i.e., there are no other major concurrent cognitive impairments) and progressive. As predicted by an amodal account, patients with semantic dementia provide impoverished concept definitions in response to pictures as well as words; they perform poorly on both picture- and word-based assessments of associative semantics; they have impaired recognition of characteristic environmental sounds and smells; and they reveal impairments in object use to an extent consistent with the degree of their semantic impairment. In addition, the patients demonstrate significant and strong item-specific associations across testing modalities, again supporting the unitary position.

Although impaired across all these assessments, the patients often exhibit significant differences between modalities. These are, however, predicted by the impact expected from the variation in mapping between surface form and meaning. For example, both words and sounds have an arbitrary relationship with meaning and the patients' performance is correspondingly poor. In contrast the more systematic relationship between form and meaning for objects and pictures leads to better performance. Some recent results from patients and computational models also indicate that conceptual representations are dynamic, i.e., they vary according to the context in which concepts are activated. For example, word definitions can be significantly improved if the word input is accompanied by a semantically related context picture, and object naming is significantly enhanced while the patients are demonstrating use of the objects. All these results are discussed with respect to contemporary models of semantic memory. 